\title{
Graphene with structure-induced spin-orbit coupling: Spin-polarized states, spin zero modes, and quantum Hall effect
}

\section{Citation}

Rashba, Emmanuel I. 2009. “Graphene with Structure-Induced Spin-Orbit Coupling: SpinPolarized States, Spin Zero Modes, and Quantum Hall Effect." Physical Review B 79 (16) (April 29). doi:10.1103/physrevb.79.161409.

\section{Published Version}

doi:10.1103/physrevb.79.161409

\section{Permanent link}

http://nrs.harvard.edu/urn-3:HUL.InstRepos:24081354

\section{Terms of Use}

This article was downloaded from Harvard University's DASH repository, and is made available under the terms and conditions applicable to Other Posted Material, as set forth at http:// nrs.harvard.edu/urn-3:HUL.InstRepos:dash.current.terms-of-use\#LAA

\section{Share Your Story}

The Harvard community has made this article openly available.

Please share how this access benefits you. Submit a story.

\section{Accessibility}




\title{
gु \\ Graphene with structure-induced spin-orbit coupling: Spin-polarized states, spin zero modes, and quantum Hall effect
}

\author{
Emmanuel I. Rashba \\ Department of Physics and Center for Nanoscale Systems, Harvard University, Cambridge, Massachusetts 02138, USA \\ and Department of Physics, Loughborough University, Leicestershire LE11 3TU, United Kingdom \\ (Received 21 February 2009; revised manuscript received 13 April 2009; published 29 April 2009)
}

\begin{abstract}
Spin splitting of the energy spectrum of single-layer graphene on $\mathrm{Au} / \mathrm{Ni}(111)$ substrate has been recently reported. I show that eigenstates of spin-orbit coupled graphene are polarized in-plane and are perpendicular to electron momentum $\mathbf{k}$; the magnitude of spin polarization $\mathbf{S}$ vanishes when $k \rightarrow 0$. In a perpendicular magnetic field $\mathbf{B}, \mathbf{S}$ is parallel to $\mathbf{B}$, and two zero modes emerge in the Landau level spectrum. Singular $\mathbf{B}$ dependence of their magnetization suggests existence of a new variety of magnetic instability. They also manifest themselves in a new variety of unconventional quantum Hall effect.
\end{abstract}

DOI: 10.1103/PhysRevB.79.161409

PACS number(s): 73.61.Wp, 73.43.-f, 71.70.Ej, 75.70.Cn

Since the discovery of graphene with its quasirelativistic energy spectrum of zero-gap Dirac fermions $s^{1,2}$ and unconventional quantum Hall effect (QHE) in single- and bilayer graphene, ${ }^{3-5}$ this material attracts attention because of its unique electronic properties and prospective applications in nanoelectronics. ${ }^{6,7}$ Applications in spintronics depend strongly on the control of spin-orbit (SO) coupling. This coupling in graphene comprises intrinsic and extrinsic components. ${ }^{8} 9$ First one is known to be very weak in free plane graphene, ${ }^{10,11}$ in carbon nanotubes it is due to their curvature. ${ }^{12-14}$ Extrinsic SO coupling originates from the interface between graphene and substrate. Recently Varykhalov et al. ${ }^{15}$ reported spin angle-resolved photoemission spectroscopy (SARPES) data from single-layer graphene on $\mathrm{Ni}(111)$ intercalated with a Au monolayer. They reveal strong momentum-dependent in-plane spin polarization. The monolayer of Au maintains the symmetry of graphene and strongly reduces the threefold deformation of graphene (originating from its coupling to $\mathrm{Ni}$ substrate) and nonequivalence of $A(B)$ lattice sites resulting from it. According to Ref. 15, the technique brings the system closer to ideal freestanding graphene than any other preparation on a solid substrate before. These results call for a systematic theory of spin polarization in freestanding graphene with extrinsic SO coupling, and in this Rapid Communication I provide such a theory for free electrons and electrons in a quantizing magnetic field $\mathbf{B}$. The results are in general agreement with the data of Varykhalov et al. ${ }^{15}$ and predict (i) dependence of spin polarization on the magnitude of the momentum $\mathbf{k}$ at the SO momentum scale, (ii) spin zero modes in a quantizing field indicating a new variety of magnetic instability, and (iii) a new variety of unconventional QHE.

A $4 \times 4$ Hamiltonian of graphene with extrinsic SO coupling can be represented in terms of a Kronecker product of $2 \times 2$ matrices $\boldsymbol{\sigma}=\left(\sigma_{1}, \sigma_{2}\right)$ and $\mathbf{s}$ as ${ }^{9}$

$$
\mathcal{H}_{K}^{0}=\gamma(\boldsymbol{\sigma} \cdot \mathbf{k})+\frac{1}{2} \lambda(\boldsymbol{\sigma} \times \mathbf{s})_{z} .
$$

Here $\gamma=\hbar v, v \approx 10^{8} \mathrm{~cm} \mathrm{~s}^{-1}$, and $\lambda$ is a SO coupling constant; for graphene/Au/Ni(111), $|\lambda| \approx 13 \mathrm{meV} .{ }^{15}$ Pauli matrices of pseudospin $\boldsymbol{\sigma}$ operate on $A(B)$ lattice cites, and $\mathbf{s}$ are
Pauli matrices for real spin. It is seen from first term in Eq. (1) that $\boldsymbol{\sigma}$ transforms as momentum $\mathbf{k}$. Therefore, as was pointed out by Kane and Mele, ${ }^{9} \mathrm{SO}$ term can be considered as a modified Rashba term with $\mathbf{k} \rightarrow \boldsymbol{\sigma}$; the conventional Rashba term $(\mathbf{k} \times \mathbf{s})_{z}$ is small and will be disregarded. In the same representation, $4 \times 4$ spin matrices are $\sigma_{0} \mathbf{s}$, with $\sigma_{0}$ for a unit matrix in $\boldsymbol{\sigma}$ space. The Hamiltonian $\mathcal{H}_{K}^{0}$ is acting near the $K$ point of the Brillouin zone (Fig. 1); for $K^{\prime}$ point, $\mathcal{H}_{K^{\prime}}^{0}=\sigma_{2} \mathcal{H}_{K}^{0} \sigma_{2}^{-1}$.

It is convenient to change from the $4 \times 4$ to a $2 \times 2$ formalism. First, we perform a unitary transformation of the Hamiltonian, $\mathcal{H}_{K}=U \mathcal{H}_{K}^{0} U^{-1}$ and of spin matrices $\sigma_{0} \mathbf{s}$ with a unitary matrix $U=\left[\left(s_{0}+s_{z}\right) \sigma_{0}+\left(s_{0}-s_{z}\right) \sigma_{1}\right] / 2$, with $s_{0}$ being a unit matrix in spin space. Then

$$
\mathcal{H}_{K}=\left(\begin{array}{cc}
0 & C \\
C^{+} & \lambda s_{y}
\end{array}\right), \quad \hat{\mathbf{S}}=\sigma_{1} \mathbf{s}, \quad \hat{S}_{z}=\sigma_{0} s_{z},
$$

with $C=\gamma\left(s_{0} k_{x}-i s_{z} k_{y}\right)$; here $\left(\hat{\mathbf{S}}, \hat{S}_{z}\right)$ are new spin matrices. Next step is eliminating lower components of eigenbispinors $\Psi=\left(\begin{array}{c}\varphi \\ \phi\end{array}\right)$ of the equation $\mathcal{H}_{K} \Psi=\varepsilon \Psi$,

$$
\phi=\left(\varepsilon-\lambda s_{y}\right)^{-1} C^{+} \varphi=\frac{\gamma}{\varepsilon^{2}-\lambda^{2}}\left(\begin{array}{cc}
\varepsilon k_{+} & -i \lambda k_{-} \\
i \lambda k_{+} & \varepsilon k_{-}
\end{array}\right) \varphi .
$$

Then we arrive at a $2 \times 2$ Hamiltonian,

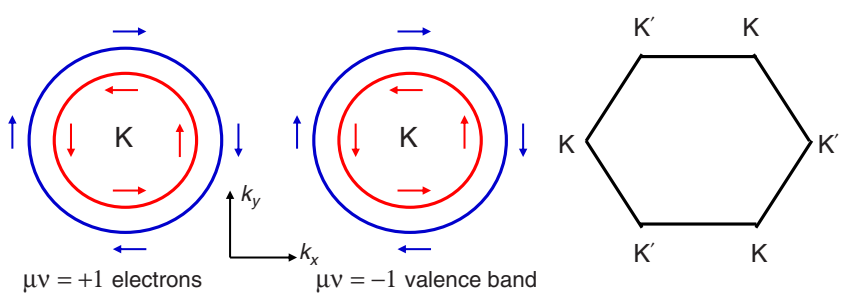

FIG. 1. (Color online) Spin polarization of the energy spectrum of spin-orbit coupled single-layer graphene with the dispersion law of Eq. (6) and spin-orbit coupling constant $\lambda>0$; energy $|\varepsilon|>\lambda$. Polarization is identical in $K$ and $K^{\prime}$ valleys. External circles: $\mu=+1$; internal circles: $\mu=-1$. For $\lambda<0$, the quantum numbers, $\mu$ and $\nu$, and spin polarizations change their signs for all branches. Brillouin zone of graphene is also shown. 


$$
H(\varepsilon)=\frac{\gamma^{2}}{\varepsilon^{2}-\lambda^{2}}\left(\begin{array}{cc}
\varepsilon k_{-} k_{+} & -i \lambda k_{-}^{2} \\
i \lambda k_{+}^{2} & \varepsilon k_{+} k_{-}
\end{array}\right),
$$

that depends explicitly on its eigenvalues $\varepsilon, H(\varepsilon) \varphi=\varepsilon \varphi$. When $\mathbf{B} \neq 0$, operators $k_{ \pm}=k_{x} \pm i k_{y}$ do not commute; below, $\mathbf{B}=B \hat{\mathbf{z}}$. We note that Eq. (4) is exact, and despite the fact that the problem is nonlinear in $\varepsilon$, calculation of spin polarization in this representation is more concise than in the original $4 \times 4$ representation.

For free carriers, $\mathbf{B}=0$, eigenspinors of $H(\varepsilon)$ are

$$
\varphi_{\nu}(\mathbf{k})=\frac{1}{\sqrt{2}}\left(\begin{array}{c}
i \nu k_{-}^{2} / k^{2} \\
1
\end{array}\right), \quad \nu= \pm 1 \text {. }
$$

From Eqs. (2) and (4) follows an equation $\varepsilon_{\nu}\left(\varepsilon_{\nu}+\lambda \nu\right)$ $=\gamma^{2} k^{2}$ for the eigenvalues $\varepsilon_{\nu}$. Its solutions are

$$
\varepsilon_{\nu \mu}(k)=\frac{\nu \mu}{2}\left(\sqrt{\lambda^{2}+4 \gamma^{2} k^{2}}-\mu \lambda\right), \quad \mu= \pm 1 .
$$

The spectrum includes two zero-gap branches and two gapped branches of the same shape. The gap equals $2|\lambda|$, and the separation between gapped and ungapped branches is $k$ independent and equals $\lambda$. The spectrum is similar to the spectrum of unbiased bilayer graphene without SO coupling, ${ }^{16-18}$ but the nature of eigenstates is quite different.

It is easily seen from Eqs. (2) and (5) that $\left\langle\varphi_{\nu}\left|\hat{S}_{z}\right| \varphi_{\nu}\right\rangle=0$; hence, spins are in-plane polarized. Because $\hat{\mathbf{S}}$ includes $\sigma_{1}$, calculation of in-plane polarization involves the lower spinor $\phi$ and is more cumbersome. Nevertheless, it is straightforward, and applying Eqs. (3), (5), and (6) results in in-plane spin polarizations $\mathbf{S}_{\nu \mu}(\mathbf{k})$ for all $(\nu, \mu)$ states,

$$
\mathbf{S}_{\nu \mu}(\mathbf{k})=\frac{\left\langle\Psi_{\nu \mu}|\hat{\mathbf{S}}| \Psi_{\nu \mu}\right\rangle}{\left\langle\Psi_{\nu \mu} \mid \Psi_{\nu \mu}\right\rangle}=\frac{2 \mu \gamma(\mathbf{k} \times \hat{\mathbf{z}})}{\sqrt{\lambda^{2}+4 \gamma^{2} k^{2}}},
$$

proportional to the group velocity $\mathbf{v}_{\nu \mu}(\mathbf{k})=\partial \varepsilon_{\nu \mu} / \hbar \partial \mathbf{k}$. Equation (7) indicates transverse spin polarization (Fig. 1), as concluded by Varykhalov et al. ${ }^{15}$ Its magnitude is $k$ dependent. When $k \gg k_{\lambda}$, with $k_{\lambda}=|\lambda| / 2 \gamma$ being a characteristic SO momentum, it saturates, $\left|\mathbf{S}_{\nu \mu}\right| \rightarrow 1$. In the $k \ll k_{\lambda}$ limit, it vanishes as $k / k_{\lambda}$. Vanishing of all $\mathbf{S}_{\nu \mu}(\mathbf{k} \rightarrow 0)$, with $\hat{\mathbf{S}}^{2}=3$, is a unique property of the Dirac points.

Chirality of the spinor $\varphi_{\nu}(\mathbf{k})$ is defined by $\nu$, spin polarization $\mathbf{S}_{\nu \mu}$ by $\mu$, and the product $\mu \nu$ specifies electron and hole spectrum branches. Experiments of Ref. 15 measured the magnitude of $\lambda,|\lambda| \approx 13 \mathrm{meV}$. Measuring the sign of $\mathbf{S}_{\nu \mu}$ would allow finding the sign of $\lambda .{ }^{19}$ Indeed, it is seen from Eq. (6) that $\mu / \lambda>0$ for external Fermi circles. Due to the requirements of time-inversion symmetry, spin polarization is identical near $K$ and $K^{\prime}$ points. It is not clear currently which of the factors (experimental resolution, temperature, or staggered potential of Ni substrate) was the main obstacle for measuring SARPES spectra for $k \leqq k_{\lambda}$. However, measurement of $\mathbf{S}_{\nu \mu}$ for $k \gtrsim k_{\lambda}$ should shed additional light on the role of these factors.

Application of well-developed techniques for detecting in-plane polarization $\mathbf{S}(\mathbf{k})$, based on Kerr spectroscopy ${ }^{20-22}$ and spin-galvanic effect, ${ }^{23}$ is hampered by the conductivity of metallic substrate. Reducing its thickness to only a few monolayers or developing insulating substrates can render them proper efficacy.

For $\mathbf{B} \| \hat{\mathbf{z}}$, applying a Peierls substitution $\mathbf{k}=-i \nabla+e \mathbf{A} / \hbar c$, with $\mathbf{A}$ being a vector potential, one expresses $k_{ \pm}$in terms of Bose operators, $k_{+}=(\sqrt{2} / \ell) a^{+}, k_{-}=(\sqrt{2} / \ell) a$, and $\left[a, a^{+}\right]=1$; here, $\ell=\sqrt{c \hbar / e B}$ is a magnetic length. Then, instead of Eq. (4), one arrives at

$$
\hat{H}(\epsilon)=|\lambda| \frac{2 \Gamma^{2}}{\epsilon^{2}-1}\left(\begin{array}{cc}
\epsilon a a^{+} & -i \beta a^{2} \\
i \beta\left(a^{+}\right)^{2} & \epsilon a^{+} a
\end{array}\right),
$$

where $\beta=\lambda /|\lambda|, \epsilon=\varepsilon /|\lambda|$, and $\Gamma=\gamma / \ell|\lambda|$. The Hamiltonian $\hat{H}(\epsilon)$ depends explicitly on its eigenvalues $\epsilon$. From here on, energy $\epsilon$ is measured in units of $|\lambda|$.

Solution of the corresponding eigenspinor problem can be found in terms of oscillator eigenfunctions ${ }^{24} \psi_{n}$,

$$
\begin{gathered}
\varphi_{n}=\left(\begin{array}{c}
c_{1} \psi_{n-2} \\
c_{2} \psi_{n}
\end{array}\right), \quad \phi_{n}=\left(\begin{array}{c}
c_{3} \\
c_{4}
\end{array}\right) \psi_{n-1}, \\
\left(\begin{array}{c}
c_{3} \\
c_{4}
\end{array}\right)=\frac{\Gamma \sqrt{2}}{\epsilon^{2}-1}\left(\begin{array}{l}
\epsilon \sqrt{n-1} c_{1}-i \beta \sqrt{n} c_{2} \\
i \beta \sqrt{n-1} c_{1}+\epsilon \sqrt{n} c_{2}
\end{array}\right),
\end{gathered}
$$

for $n \geq 2$. The coefficients $c_{1,2}=c_{1,2}(n)$, normalized as $\left|c_{1}\right|^{2}+\left|c_{2}\right|^{2}=1$, read as

$$
\frac{c_{1}}{c_{2}}=\frac{i \beta \epsilon\left(1+2 n \Gamma^{2}-\epsilon^{2}\right)}{2 \sqrt{n(n-1)} \Gamma^{2}}, \quad c_{2}=\frac{\sqrt{n}}{2 n\left(1+\Gamma^{2}\right)-\epsilon^{2}} .
$$

Eigenvalues obey the equation

$$
\epsilon^{4}-\left[1+2 \Gamma^{2}(2 n-1)\right] \epsilon^{2}+4 n(n-1) \Gamma^{4}=0
$$

and are

$$
\left(\epsilon_{n}^{ \pm}\right)^{2}=\frac{1}{2}\left[1+2(2 n-1) \Gamma^{2} \pm \sqrt{1+4(2 n-1) \Gamma^{2}+4 \Gamma^{4}}\right] .
$$

Equation (12) coincides with the expression for bilayer graphene in absence of SO coupling. ${ }^{18,25,26}$

In addition to the solutions with $n \geq 2$, there are solutions with $n \leq 1$ that are of special interest because of their peculiar spin properties. For $n=0$, there is a single solution $c_{1}(0)=0, c_{2}(0)=1$, and $\phi_{0}=0$, with $\epsilon_{0}=0$. For $n=1$, there are three solutions with $c_{1}(1)=0$ and $c_{2}(1)=1$; they differ by their $\phi_{1}$ spinors. For one of them the eigenvalue vanishes, $\epsilon_{1}^{0}=0$, and components of the spinor $\phi_{1}^{0}$ are $c_{3}=i \beta \Gamma \sqrt{2}$ and $c_{4}=0$. Two nonvanishing eigenvalues are $\epsilon_{1}= \pm \sqrt{1+2 \Gamma^{2}}$; the components of the corresponding spinors $\phi_{1}$ are $c_{3}=-i \beta / \Gamma \sqrt{2}, c_{4}=\epsilon_{1} / \Gamma \sqrt{2}$. These expressions can also be found from Eqs. (9) and (12) [with the upper sign in Eq. (12)] by plugging $n=1$. Hence, there exist two zero modes, $\epsilon_{0}=0$ and $\epsilon_{1}^{0}=0$, and SO coupling of $(\boldsymbol{\sigma} \times \mathbf{s})$ type preserves twofold degeneracy of the $\epsilon=0$ state typical of single-layer graphene $e^{3,4,27}$ but changes its nature: degeneracy is dynamical rather than symmetry conditioned (small Zeeman splitting ${ }^{28}$ is disregarded).

It follows from Eq. (9) that the in-plane spin polarization $\mathbf{S}$ vanishes in all eigenstates. Indeed, because of the factor $\sigma_{1}$ in $\hat{\mathbf{S}}=\sigma_{1} \mathbf{S}$, it mixes different components of the bispinor $\Psi, \varphi$ 
and $\phi$, and the mean value of their product is proportional to scalar products of the oscillator functions $\psi_{m}$ with quantum numbers that never coincide. Therefore, $\mathbf{S}_{n}=0$ for all $n$. This result is expected because $\mathbf{S}_{\nu \mu}(\mathbf{k})$ of Eq. (7) vanishes after averaging over the direction of $\mathbf{k}$ and follows from axial symmetry of the problem.

Longitudinal polarization $S_{z}$, after eliminating $\phi$ component of $\Psi$, can be expressed in terms of its $\varphi$ component,

$$
S_{z}=\left\langle\varphi\left|s_{z}+\frac{\Gamma^{2}}{\epsilon^{2}-1}\left[s_{0}+\left(a a^{+}+a^{+} a\right) s_{z}\right]\right| \varphi\right\rangle /\langle\Psi \mid \Psi\rangle
$$

$\epsilon$ is the energy of the state $\varphi$, and normalization factor

$$
\begin{aligned}
\langle\Psi \mid \Psi\rangle= & 1+\frac{\Gamma^{2}}{\left(\epsilon^{2}-1\right)^{2}}\langle\varphi| 2 i \beta\left[\left(a^{+}\right)^{2} s_{-}-a^{2} s_{+}\right] \\
& +\left(\epsilon^{2}+1\right)\left[\left(a a^{+}+a^{+} a\right) s_{0}+s_{z}\right]|\varphi\rangle .
\end{aligned}
$$

Explicit expressions for Eqs. (13) and (14) follow from Eqs. (9) and (10). Their original form is cumbersome but greatly simplifies after higher powers of $\epsilon^{2}$ are eliminated by employing Eq. (11), and $\beta^{2}=1$ is applied. The final equation, when expressed in terms of $\epsilon_{n}^{ \pm}$, is rather concise,

$$
\left(S_{z}\right)_{n}^{ \pm}=\frac{2 \Gamma^{2}\left(2 n \Gamma^{2}-\epsilon^{2}\right)}{\epsilon^{2}\left(1-2 \Gamma^{2}\right)+2 n \Gamma^{2}\left(1+2 \Gamma^{2}\right)}, \quad \epsilon=\epsilon_{n}^{ \pm} .
$$

This equation, together with Eq. (12), provides exact expressions for $B$ dependence of spin polarization for all states with $n \geq 1$ and $\epsilon \neq 0$. Because $\epsilon$ appears in Eq. (15) only as $\epsilon^{2}$, polarization $S_{z}$ is charge symmetrical: it coincides for electron and hole states with the same $n$ and $\left|\epsilon_{n}^{ \pm}\right|$.

In the weak field limit, $n \Gamma^{2} \ll 1, \epsilon^{2}$, eigenvalues are $\epsilon_{n}^{+} \approx 1$ and $\epsilon_{n}^{-} \approx 2 \sqrt{n(n-1)} \Gamma^{2}$, and $S_{z}$ is $n$ independent and proportional to $B,\left(S_{z}\right)_{n}^{ \pm} \approx \mp 2 \Gamma^{2}$ ( $n>1$ for $\epsilon_{n}^{-}$states). Because of the spectrum degeneracy at the $k=0$ point, the sequence $\epsilon_{n}^{-}$is nonequidistant despite the parabolicity of the spectrum. ${ }^{24}$ In the strong field limit, $\Gamma^{2} \rightarrow \infty$, eigenvalues are $\epsilon_{n}^{-} \approx \Gamma \sqrt{2 n}$ and $\epsilon_{n}^{+} \approx \Gamma \sqrt{2(n-1)}$, and spin magnetization saturates, $\left(S_{z}\right)_{n}^{ \pm} \approx \mp 1$. In this limit, two ladders nearly overlap and are split by $\Delta \epsilon_{n} \approx \sqrt{n / 2} /(2 \Gamma)$; the splitting increases with $n$ but for $\Gamma^{2} \gg n$ is small compared with the level separation $\Gamma / \sqrt{2 n}$ inside each ladder. For $\lambda \approx 13 \mathrm{meV}$, the field separating these two limit cases, found from the condition $\Gamma=1$, equals $B_{\mathrm{cr}}=c \lambda^{2} /\left(e \hbar v^{2}\right) \approx 0.3 \mathrm{~T}$.

In both limits, spin magnetization has opposite sign for $\epsilon^{+}$ and $\epsilon^{-}$ladders; hence, magnetization oscillates when Landau levels cross the Fermi level. These de-Haas-van-Alphen-type oscillations can be detected by Kerr spectroscopy ${ }^{29,30}$ even for ferromagnetic substrates, and by torque magnetometer techniques. $^{31}$

Semiclassical regime is achieved for $\Gamma \ll 1$ and $n \gg 1$ with $n \Gamma^{2}=\kappa^{2} / 2=$ const; for $\kappa=\gamma k /|\lambda|$, one recovers Eq. (6) from Eq. (12). Keeping $\kappa=$ const, one finds for electron branches, $\epsilon_{n}^{ \pm}>0$, in the first order in $1 / n$,

$$
\epsilon_{n}^{ \pm} \approx \frac{1}{2}\left(\sqrt{1+4 \kappa^{2}} \pm 1\right)-\frac{\kappa^{2}}{2 n \sqrt{1+4 \kappa^{2}}},
$$

$$
\left(S_{z}\right)_{n}^{ \pm} \approx \mp \frac{\kappa^{2}}{n \sqrt{1+4 \kappa^{2}}}=\mp \frac{2 \Gamma^{2}}{\sqrt{1+4(\gamma k / \lambda)^{2}}} .
$$

Therefore, in the leading order of the expansion, level splitting $\Delta \epsilon_{n} \approx 1$ remains $n$ independent, while spin polarization $S_{z}$ has opposite sign for two spectrum branches and decreases with $n$ (or, for $\Gamma=$ const, with the electron momentum $k$ ). The oscillatory dependence of the total spin magnetization on $B$ can be detected as discussed in the previous paragraph.

Therefore, all quantum states with $\epsilon_{n}^{ \pm} \neq 0$ are nondegenerate, and only two states $\epsilon_{0}=\epsilon_{1}^{0}=0$ are degenerate. These two states differ strongly in their spin magnetization. For the $n=0$ state, polarization $\left(S_{z}\right)_{0}=-1$. It does not depend on $B$ but changes abruptly when $\mathbf{B}$ changes sign. For the $n=1$ state with $\epsilon_{1}^{0}=0$, polarization equals $\left(S_{z}\right)_{1}=-\left(1-2 \Gamma^{2}\right) /\left(1+2 \Gamma^{2}\right)$; it tends to -1 for $B \rightarrow 0$, changes sign at $\Gamma^{2}=1 / 2$, and saturates to +1 for $B \rightarrow \infty$. In this limit, contributions of two $\epsilon=0$ states cancel. Such a behavior is unique because it suggests that SO coupled graphene with filled $\epsilon=0$ states is unstable to ferromagnetic ordering in $\hat{\mathbf{z}}$ direction in weak fields $\mathbf{B} \| \hat{\mathbf{z}}$, while with increasing $B$ the magnetization gradually vanishes. This magnetization differs drastically from the edge-state magnetization of graphene zigzag nanoribbons proposed by Fujita et $a .^{32}$ because it originates from SO coupling rather than from the exchange interaction. It also bears no similarity with the Dzyaloshinskii-Moriya weak ferromagnetism ${ }^{33,34}$ because it is dynamical rather than symmetry conditioned and develops in a paramagnet without any magnetic structure. The effect of electron-electron interaction on this peculiar state needs a special investigation. ${ }^{35}$

For comparison with experimental data, one needs to add magnetization $S_{z}$ of the electrons in $K$ and $K^{\prime}$ valleys. Because $\mathcal{H}_{K^{\prime}}^{0}$ is related to $\mathcal{H}_{K}^{0}$ by a $\sigma_{2}$ canonical transformation leaving the operator $\hat{S}_{z}$ of Eq. (2) unchanged, magnetization has the same magnitude and sign in both valleys. This can be also inferred from the time-inversion symmetry requirements. Therefore, total $S_{z}$ equals the magnetization of the $K$ valley multiplied by the factor of 2 .

Novoselov et al. ${ }^{5}$ compared the conventional QHE (Ref. 36) and two types of unconventional QHEs typical of single-layer ${ }^{3,4}$ and bilayer graphene, the material notorious for its exotic QHE properties. ${ }^{37}$ SO coupled single-layer graphene introduces one more type of unconventional QHE. Due to the fourfold degeneracy of zero modes $\left[\epsilon_{0}=\epsilon_{1}^{0}=0\right.$ degeneracy times the factor of 2 from isospin, $K\left(K^{\prime}\right)$ valleys] the step in $\sigma_{x y}$ at $B=0$ equals $4 e^{2} / h$, as in single-layer graphene without SO coupling. However, because spin degeneracy is lifted in each valley by SO interaction, and only isospin degeneracy persists, all $B \neq 0$ steps are of $2 e^{2} / h$. Therefore, the ratio of the magnitudes of the $B=0$ and $B \neq 0$ steps equals 2 , as in bilayer graphene without $\mathrm{SO}$ coupling. Depending on the magnitude of $\lambda$, these $2 e^{2} / h$ steps can appear in pairs, as resolved $4 e^{2} / h$ steps, similar to the resolution of two spin components of the traditional QHE. Spin-orbit coupled graphene on an isolating substrate would become the optimal object for observing this new variety of QHE and for spin manipulation, a challenging task for semiconductor spintronics. 
Apparently, among the perturbations that lower the symmetry of the Hamiltonian the largest is the staggering sublattice potential of the substrate violating the equivalence of $A(B)$ lattice cites; manifestation of the broken sixfold symmetry was reported in Ref. 15. A staggered potential can be described by a term $\mathcal{H}_{\mathrm{st}}=u \sigma_{z} \tau_{z}$, where $\tau_{z}$ is a Pauli matrix in the isospin space. ${ }^{9}$ This term creates a gap in the spectrum and lifts the symmetry of $K\left(K^{\prime}\right)$ valleys. Adding $\mathcal{H}_{\text {st }}$ to $\mathcal{H}_{K\left(K^{\prime}\right)}^{0}$ does not change wave functions of $n=0$ modes but changes their energies to $\varepsilon= \pm u$ (in dimensional units). For the $n=1$ soft mode, eigenvectors also change; now $c_{1}=0$ but $c_{2}, c_{3}, c_{4} \neq 0$. Energy spectrum can be found from a cubic equation that for $|u / \lambda| \ll 1$ defines soft modes $\varepsilon_{1} \approx \pm u\left(1-2 \Gamma^{2}\right) /\left(1+2 \Gamma^{2}\right)$. As a result, the $4 e^{2} / h$ step in $\sigma_{x y}$ splits into a plateau near $B=0$ and two $e^{2} / h$ steps on both sides of it. Lifting the $K\left(K^{\prime}\right)$ degeneracy also splits all $2 e^{2} / h$ steps, making the QHE of SO coupled graphene similar to the traditional QHE with a resolved Zeeman splitting. We notice that $\lambda=13 \mathrm{meV}$ corresponds to a field $B=120 \mathrm{~T}$ for a Landé factor $g=2$.

In conclusion, a theory of the energy spectrum and spin polarization in single-layer graphene, a subject to a substrateinduced spin-orbit coupling, is presented. Energy spectrum consists of two zero-gap bands and two gapped bands, and all states are in-plane spin-polarized perpendicular to the momentum k. This polarization saturates at large $k$ and vanishes at the scale of spin-orbit energy when $k \rightarrow 0$. In a perpendicular magnetic field, two zero modes develop in each of $K\left(K^{\prime}\right)$ valleys. These modes show a peculiar magnetic behavior suggesting a possibility of a perpendicular-to-plane spinorbit conditioned magnetism and produce a new variety of unconventional quantum Hall effect.

I am grateful to F. Kuemmeth, C. M. Marcus, J. R. Williams, and R. Winkler for stimulating discussions.
${ }^{1}$ K. S. Novoselov, A. K. Geim, S. V. Morozov, D. Jiang, Y. Zhang, S. V. Dubonos, I. V. Grigorieva, and A. A. Firsov, Science 306, 666 (2004).

${ }^{2}$ P. R. Wallace, Phys. Rev. 71, 622 (1947).

${ }^{3}$ K. S. Novoselov, A. K. Geim, S. V. Morozov, D. Jiang, M. I. Katsnelson, I. V. Grigorieva, S. V. Dubonos, and A. A. Firsov, Nature (London) 438, 197 (2005).

${ }^{4}$ Y. Zhang, Y.-W. Tan, H. L. Stormer, and P. Kim, Nature (London) 438, 201 (2005).

${ }^{5}$ K. S. Novoselov, E. McCann, S. V. Morozov, V. I. Fal'ko, M. I. Katsnelson, U. Zeitler, D. Jiang, F. Schedin, and A. K. Geim, Nat. Phys. 2, 177 (2006).

${ }^{6}$ C. W. J. Beenakker, Rev. Mod. Phys. 80, 1337 (2008).

${ }^{7}$ A. H. Castro Neto, F. Guinea, N. M. R. Peres, K. S. Novoselov, and A. K. Geim, Rev. Mod. Phys. 81, 109 (2009).

${ }^{8}$ G. Dresselhaus and M. S. Dressehaus, Phys. Rev. 140, A401 (1965).

${ }^{9}$ C. L. Kane and E. J. Mele, Phys. Rev. Lett. 95, 226801 (2005).

${ }^{10}$ H. Min, J. E. Hill, N. A. Sinitsyn, B. R. Sahu, L. Kleinman, and A. H. MacDonald, Phys. Rev. B 74, 165310 (2006).

${ }^{11}$ Y. Yao, F. Ye, X. L. Qi, S. C. Zhang, and Z. Fang, Phys. Rev. B 75, 041401(R) (2007)

${ }^{12}$ T. Ando, J. Phys. Soc. Jpn. 69, 1757 (2000).

${ }^{13}$ D. Huertas-Hernando, F. Guinea, and A. Brataas, Phys. Rev. B 74, 155426 (2006).

${ }^{14}$ F. Kuemmeth, S. Ilani, D. C. Ralph, and P. L. McEuen, Nature (London) 452, 448 (2008).

${ }^{15}$ A. Varykhalov, J. Sanchez-Barriga, A. M. Shikin, C. Biswas, E. Vescovo, A. Rybkin, D. Marchenko, and O. Rader, Phys. Rev. Lett. 101, 157601 (2008).

${ }^{16}$ E. McCann and V. I. Fal'ko, Phys. Rev. Lett. 96, 086805 (2006).

${ }^{17}$ J. Nilsson, A. H. Castro Neto, F. Guinea, and N. M. R. Peres, Phys. Rev. Lett. 97, 266801 (2006).

${ }^{18}$ J. M. Pereira, Jr., F. M. Peeters, and P. Vasilopoulos, Phys. Rev. B 76, 115419 (2007).

${ }^{19}$ J. Henk, M. Hoesch, J. Osterwalder, A. Ernst, and P. Bruno, J. Phys.: Condens. Matter 16, 7581 (2004).
${ }^{20}$ Y. Kato, R. C. Myers, A. C. Gossard, and D. D. Awschalom, Nature (London) 427, 50 (2004).

${ }^{21}$ S. A. Crooker and D. L. Smith, Phys. Rev. Lett. 94, 236601 (2005).

${ }^{22}$ L. Meier, G. Salis, I. Shorubalko, E. Gini, S. Schön, and K. Ensslin, Nat. Phys. 4, 77 (2008).

${ }^{23}$ E. L. Ivchenko and S. Ganichev, Spin Physics in Semiconductors (Springer, New York, 2008), p. 245.

${ }^{24}$ J. M. Luttinger, Phys. Rev. 102, 1030 (1956).

${ }^{25}$ M. Nakamura, L. Hirasawa, and K.-I. Imura, Phys. Rev. B 78, 033403 (2008).

${ }^{26}$ E. A. Henriksen, Z. Jiang, L. C. Tung, M. E. Schwartz, M. Takita, Y. J. Wang, P. Kim, and H. L. Stormer, Phys. Rev. Lett. 100, 087403 (2008).

${ }^{27}$ V. P. Gusynin and S. G. Sharapov, Phys. Rev. Lett. 95, 146801 (2005).

${ }^{28}$ Y. Zhang, Z. Jiang, J. P. Small, M. S. Purewal, Y. W. Tan, M. Fazlollahi, J. D. Chudow, J. A. Jaszczak, H. L. Stormer, and P. Kim, Phys. Rev. Lett. 96, 136806 (2006).

${ }^{29}$ D. D. Awschalom and J. M. Kikkawa, Phys. Today 52 (6), 33 (1999).

${ }^{30}$ J. Xia, E. Schemm, G. Deutscher, S. A. Kivelson, D. A. Bonn, W. N. Hardy, R. Liang, W. Siemons, G. Koster, M. M. Fejer, and A. Kapitulnik, Phys. Rev. Lett. 100, 127002 (2008).

${ }^{31}$ M. A. Wilde, D. Reuter, Ch. Heyn, A. D. Wieck, and D. Grundler, Phys. Rev. B 79, 125330 (2009), and references therein.

${ }^{32}$ M. Fujita, K. Wakabayashi, K. Nakada, and K. Kusakabe, J. Phys. Soc. Jpn. 65, 1920 (1996).

${ }^{33}$ I. E. Dzialoshinskii, Sov. Phys. JETP 5, 1259 (1957).

${ }^{34}$ T. Moriya, Phys. Rev. 120, 91 (1960).

${ }^{35}$ K. Nomura and A. H. MacDonald, Phys. Rev. Lett. 96, 256602 (2006).

${ }^{36}$ K. v. Klitzing, G. Dorda, and M. Pepper, Phys. Rev. Lett. 45, 494 (1980).

${ }^{37}$ F. D. M. Haldane, Phys. Rev. Lett. 93, 206602 (2004). 